\title{
Marijuana use and cervical HPV/neoplasia
}

G D'Souza*1, R Burk², Y Zhong², H Minkoff³, LS Massad4, K Anastos, A Levine ${ }^{6}, \mathrm{M} \mathrm{Moxley}^{7}, \mathrm{X} \mathrm{Xue}^{5}$, J Palefsky ${ }^{8}$ and HD Strickler ${ }^{2}$

Address: ${ }^{1}$ Department of Epidemiology, Johns Hopkins Bloomberg School of Public Health, Baltimore, Maryland, USA, ${ }^{2}$ Department of Epidemiology \& Social Medicine, Albert Einstein College of Medicine, Bronx, New York, USA, ${ }^{3}$ Maimonides Medical Center and SUNY Downstate, Brooklyn, New York, USA, ${ }^{4}$ Southern Illinois University School of Medicine, Springfield, Illinois, USA, ${ }^{5}$ Albert Einstein College of Medicine, Bronx, New York, USA, ${ }^{6}$ University of Southern California, Los Angeles, California, USA, ${ }^{7}$ Georgetown University Medical Center, Washington, DC, USA and ${ }^{8}$ University of California at San Francisco, California, USA

* Corresponding author

from I I th International Conference on Malignancies in AIDS and Other Acquired Immunodeficiencies (ICMAOI): Basic, Epidemiologic, and Clinical Research

Bethesda, MD, USA. 6-7 October 2008

Published: 17 June 2009

Infectious Agents and Cancer 2009, 4(Suppl 2):PI5 doi:I0.II86/I750-9378-4-S2-PI5

This abstract is available from: http://www.infectagentscancer.com/content/4/S2/PI5

(c) 2009 D'Souza et al; licensee BioMed Central Ltd.

Recent research suggests that marijuana use may be positively associated with risk of human papillomavirus (HPV)-associated oropharyngeal cancer, and the immunomodulatory effects of cannabinoids are considered a possible mechanism. Were marijuana to have systemic effects on HPV-associated tumorigenesis it would be of major concern, and might be especially harmful in immunologically susceptible populations such as HIV-positive women.

We studied the effect of marijuana use on cervical HPV natural history and cervical squamous intraepithelial lesions (SIL) among HIV-positive and HIV-negative women in the Women's Interagency HIV Study (WIHS), a large prospective cohort study. HPV DNA testing by PCR and Pap smears were conducted semi-annually. Prevalent HPV and cervical SIL were analyzed using logistic regression with GEE. Persistence of HPV was analyzed using Cox regression.

Of the 3,499 women in the WIHS, 1,414 reported ever using marijuana. There were no associations between HPV prevalence and either current $(\mathrm{OR}=0.9895 \% \mathrm{CI}=$ 0.90-1.07), frequent (current daily use: $\mathrm{OR}=0.9895 \mathrm{CI}$ $=0.90-1.07$ ), or sustained (daily use for more than 3 years: $\mathrm{OR}=1.03,95 \% \mathrm{CI}=0.90-1.19)$ marijuana use, controlling for age, race, HIV status, CD4 cell count, number of sexual partners in the last 6 months, tobacco use and cervical treatment. Nor were there effects on prevalence of SIL, or on the persistence of cervical HPV infection. Results were similar among HIV-negative and HIVpositive women.

Our data do not support an effect of marijuana on cervical HPV infection, persistence or risk of cervical dysplasia. If there is an effect of marijuana use on oral HPV-associated tumorigenesis, it may be a local effect. 